\title{
Digitization: Creative and Open-ended
}

\section{Authors}

Dan C. Baciu ${ }^{1}$

Delft University of Technology, Netherlands.

\begin{abstract}
The alphabet, the genetic code, and all of our human languages and those used by computers have something in common: They all are examples of digital systems. The main mathematical property of digital systems is that they use only a small number of symbols to create open-ended worlds of possibilities. For example, by creatively combining and recombining the fingers on one hand, digital systems can encode for countless numbers. One can be creative, count, and count, and never return to the same number. In digital systems, there typically are so many possibilities to explore that diversification becomes necessary to cover sufficient ground. Thus, creativity and diversification are consequences of digital systems. In this article, I explain how these two processes - creativity and diversification - can be modeled with replicator-mutator-equation, and how this equation describes and explains a broad range of observations in human behavior, culture, and society.
\end{abstract}

\footnotetext{
${ }^{1}$ https://orcid.org/0000-0002-0043-5616
} 


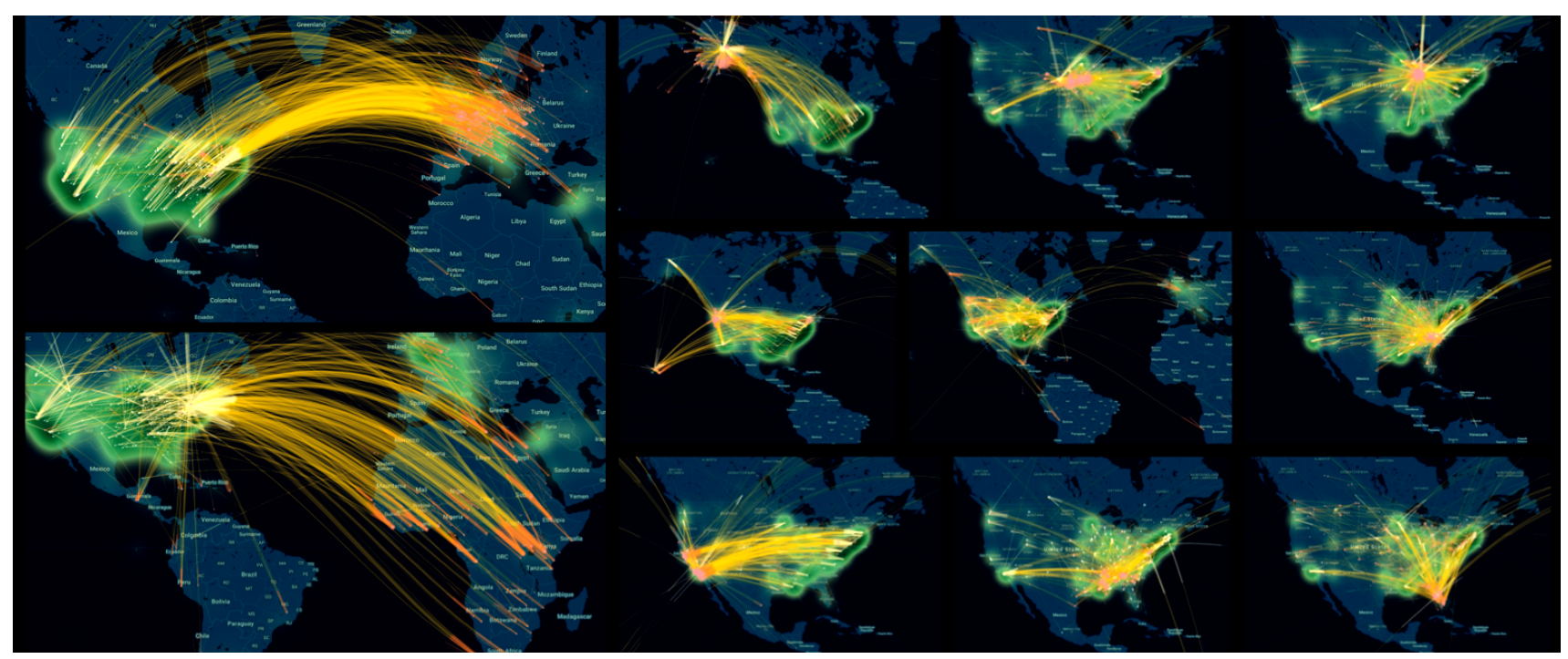

Fig. 1. A digital tool to study the geography of culture, as taken from my 2020 research paper. Each map represents a group of ideas. Source, theory, and interactive visual: https://doi.org/10.25496/W2H592

\section{What digitization is, and where it originated?}

A digital system is something quite practical, and it comes very natural to most of us. In a digital system, a small number of symbols, for example zeroes and ones, are combined and recombined to encode for a much larger, often open-ended number of other symbols. Imagine a practical application. Somebody asks you to create 1,000 distinct passwords. You can easily achieve this by combining and recombining zeros and ones. With only ten digits, you can create $2^{10}$ passwords. That's more than enough. Along the way, one thing has become evident: A digital system is a system in which symbols are combined and recombined to stunning effect.

Digital systems are used in digital computers, but not exclusively, and not even primarily. The terms "digital" and "digit" are older than any digital computer. They come from the Latin word "digitus, digiti", which means "finger, fingers." This etymology is based on a history that is straightforward: Before people used their computers to do their accounting, they counted on their fingers. Admittedly, a big change has occurred. The fingers were taught that to use computers. And certainly, computers brought a lot of speed, but they did this by keeping the digital system. Numerical systems, as the ones used to count on one's fingers, were already digital systems because a small number of symbols is combined and recombined to encode for an infinity of natural numbers.

Numerical systems and computer systems are not the only digital systems we know of. Another, equally important digital system is the alphabet. The alphabet specifies a small number of letters that can be combined and recombined to create an open-ended number of words. In turn, it is no coincidence that the alphabet is a digital system. All human languages are digital. All languages combine and recombine sounds to create an open-ended number of words; and words are recombined into sentences, stories, and into entire cultures (Nowak, Komarova 2001). All human cultures are digital cultures; they are based on symbols that are recombined towards the expression of open-ended cultural possibilities.

Of course, human cultures as they are, aided by their alphabets, numbers, and computers, have swept the globe, but human cultures are not the first wave of digitization, and not even the largest. All biological life, from viruses to multicellular organisms, is digital. All biological life is based on the genetic code. The genetic code is the same for all living beings on Earth. And, the genetic code is a digital code. Two base pairs - one could have decided to call them " 0 " and " 1 " - are combined and recombined to encode for open-ended genetic information. In this sense, all biological life on Earth is digital. Biological life probably is the largest wave of digitization, but the various waves of digitization are most powerful in unison. They are together transforming the physical world (Fig. 1). 


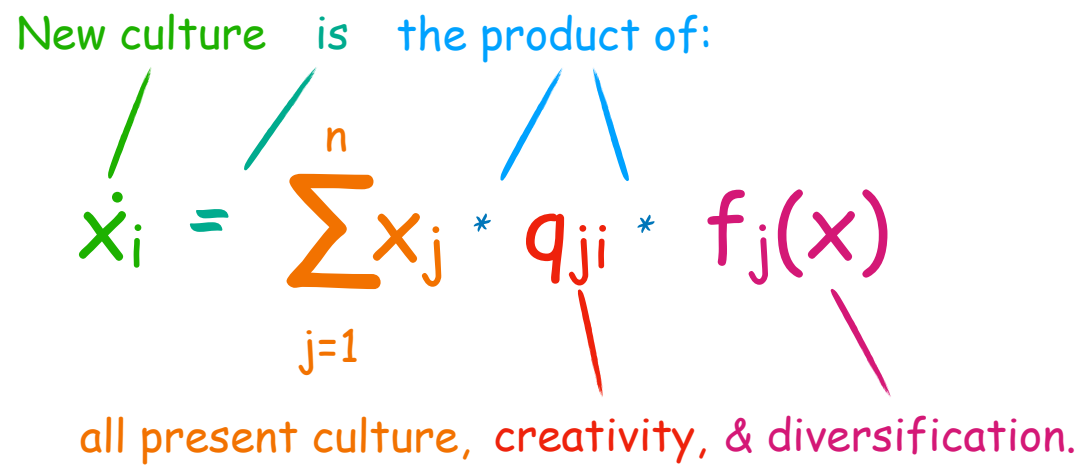

Fig. 2. Each idea in the next generation of culture is the product of all ideas in present culture, creativity, \& diversification.

If applied to the life sciences, creativity can also be understood as intra-species evolution. Diversification can also be understood as inter-species evolution, or as ecology. Technically, the term $\mathrm{q}_{\mathrm{ij}}$ stays for any creativity matrix, mutation matrix, or association matrix. This term is also used in variation-selection processes and the quasispecies equation. The term $\mathrm{f}_{\mathrm{i}}(\mathrm{x})$ stays for the interplay between diverse variants. This term is equivalent to frequency dependent selection and the replicator equation, or to generalized Lotka-Volterra equations, and it is closely related to the SIR model, as has been demonstrated by Page \& Nowak 2002. I have introduced these equations to the humanities over the course of the last decade.

\section{Creativity, open-endedness, \& the mathematics of digital systems}

The fact that almost all biological and cultural life is based on digital systems has important consequences. In all digital systems, the possibility of combining and recombining symbols opens the path to creativity. And creativity, as played out in entire populations of individuals, allows for evolutionary search and adaptation. On the other hand, open-endedness opens the path to diversification. In any open-ended system, there are so many possibilities to be explored that diversification is as good as necessary to cover any substantial ground. Diversification allows living beings to explore the open-ended possibilities of digital systems as broadly as possible.

These two processes - creativity and diversification - not only are directly linked to the most important properties of digital systems. They can be formulated as mathematical equations (Eigen 1971, Hofbauer, Sigmund 1998), and they have been united and expressed as one single equation. According to this equation, new culture is the product of old culture transformed by creativity and by diversification (Fig.2).

This mathematics is new to the humanities. I have introduced it to this field of study over the last decade (Baciu 20152021). Yet, my work has only expanded on some of the greatest achievements of modern life science. What I just called creativity is known in the life sciences as "evolution," and what I called diversification is known in the life sciences as the study of diversity in ecosystems, or, with one single word as "ecology."

Evolution and ecology are often studied through the same equations that I have introduced to the humanities. Given that both biological and cultural life is digital, the equations not only apply to biological life but also to human cultures. What life scientists call evolution may then be best termed just as I phrased it, namely as "collective creativity," and ecology may be termed as "cultural diversification."

Unsurprisingly, all human cultures know of creativity and diversity. The life science equations that I introduced to the humanities help us model these ubiquitous aspects of culture. In my research, I have developed the theory to do this, and I have provided predictions and massive empirical testing (Baciu 2017, 2018, 2020; Baciu, Della Pietra 2021).

The applications of my research are numerous. In cities, my theory sheds new light on the study of street life, urban growth, and gentrification (Fig. 3). I predict that urbanization goes through entire cycles of diversification, growth, competitive exclusion, and reform. In human culture, my theory allows to study how fashions and trends come, pass, and return. Here again, the theory explains diversification, and it predicts entire cycles of formation, growth, competitive exclusion, and reform. To bring it to the point: Growth and recession are cycles of diversification. Along the way, creativity and diversification lead us to the study of Pareto distributions and to the statistics of diversity indices. And there is more. In art and architecture, my theory explains the evolution of styles. In linguistics, it explains the evolution of diverse semantic groups, and in sociology, the evolution of diverse social fields. 


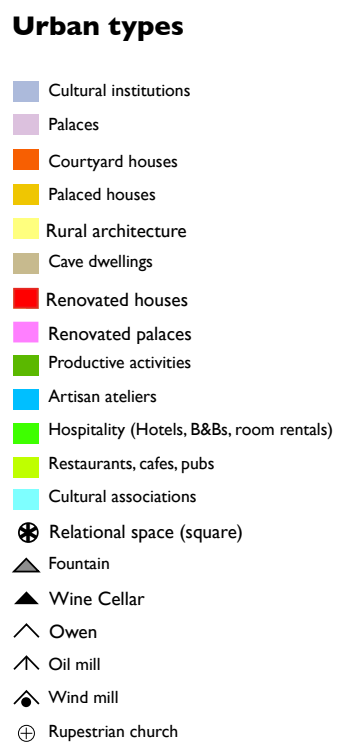

\section{Diversity analysis}

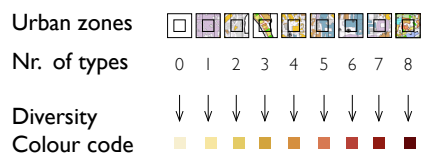

Fig. 3. A practical application of the study of diversification in cities. Quantitative evidence from the Italian UNESCO world heritage site of Sassi supports the thesis that urban environments go through cycles of diversification and growth, as modeled with the replicator-mutator-equation (Baciu, Della Pietra 2021).

And here comes the latest wave of digital tools. In the 1960s, when the sociologist Pierre Bourdieu came up with his theory of social fields, his work was based on some obscure math (Bourdieu 1966). I say "obscure" because Bourdieu's theory does not directly explain that math. Meanwhile, digital sociologists, digital humanists, digital anthropologists, computational psychologists, and many more have made modifications to this original math. Their goal was to obtain clearer and clearer empirical analyses. Over time, the math has evolved to work better and better-although, researchers sometimes disagreed which math worked best, and they mostly disagreed as of why. New theories have evolved, too, although quite independently. For example, computational psychologists dramatically broke with the past when they attempted to develop a theory of language without precedent to the $21^{\text {st }}$ century (Landauer, Dumais 1997). In my work, I have shown that the latest mathematical models used by digital humanists, sociologists, psychologists, etc. can easily be derived from the previously mentioned equations of life science, and so can be some of the most important digital tools that have been developed in the industry (for example Google's PageRank). When one looks at these tools and how they changed over the course of time, a general trend is clear: The mathematical models used by the researchers and engineers who study human culture have become increasingly akin to those used by life scientists (Wagner, Rosen 2014). As I have shown, these models are not just similar, they are mathematically equivalent. The reason behind this mathematical equivalence is the fact that all life, cultural and biological, is digital.

Ironically, the term "digital" in "digital humanist", "digital anthropologist", and the like was chosen because these digital researchers use digital computers. Who does not? "Digital" in "digital humanist" is used as in the sentence "The digital revolution first swept the United States and the Netherlands." I believe that it would be just as appropriate to understand "digital" in "digital humanist", "digital anthropologist", "digital sociologist", "digital linguist" or the like as in "Almost all biological and cultural life is digital."

This insight also means that life scientists, ecologists, epidemiologists, linguists, humanists, scholars, geographers, and many more researchers and engineers can learn from each other. Digital systems are the past, present, and future of life. They are everywhere we look. With digital systems come symbols that are combined and recombined to create open-ended worlds of possibilities, and with recombination and open-endedness come collective creativity and diversification. In turn, creativity and diversification can be studied with the same mathematics, regardless of the discipline. 


\section{Easy access to articles and datasets}

Baciu DC, Callum B (2021). "Mapping Diversity: From Ecology and Human Geography to Urbanism and Culture." OSF preprints. https://doi.org/10.31219/osf.io/sdvaz

Baciu DC (2020). "Cultural life: Theory and empirical testing." Biosystems 197.104208. Article publicly available at: https://doi.org/10.1016/j.biosystems.2020.104208

Baciu DC (2019). "Chicago Schools: Large-scale Dissemination and Reception." Prometheus 2: 20-43. https://escholarship.org/uc/item/22v9g5m

Data processed with geocodes, visualized. Publicly available: https://doi.org/10.25496/W2RP4H

Data processed with publisher names, visualized. Publicly available: https://doi.org/10. 25496/W2WC7S

Data processed with historical information, visualized. Publicly available: https://doi.org/10.254 96/W2159Q

Google ngrams (2012) publicly available: https://storage.googleapis.com/books/ngrams/books/datasetsv3.html

\section{Bibliography (including supplement)}

Baciu DC (2015). “Systemic Analysis of Cooperation: Architects, Urban Space and Tourism.” JETK 4, 27-30. Baci DC (2016). "Sigfried Giedion: Historiography and History of Reception on a Global Stage."

Ar(t)chitecture, Haifa: Technion Israel Institute of Technology, 40-52.

Baciu DC (2017). “The Chicago School: Evolving Systems of Value.” Report, HathiTrust Research Center.

Baciu DC (2018). "From Everything Called Chicago School Towards the Theory of Varieties." Doctoral dissertation, Illinois Institute of Technology.

Baciu DC (2019). "The Chicago School: Large-Scale Dissemination and Reception.” Prometheus 2, 20-41.

Baciu DC (2020). "Cultural Life: Theory and Empirical Testing." Biosystems 197.104208.

Baciu DC (2021). "Is "Cultural Life” a Form of Life?" Lecture, TU Delft, published in: Igloo Feb. 2021, 5861.

Baciu DC, Birchall C (2021). "Mapping Diversity: From Ecology and Human Geography to Urbanism and Culture." OSF preprints.

Baciu DC, Della Pietra D (2021). "Cycles of Diversification in Urban Environments." OSF preprints.

Bejan A (1996). Constructal-theory network of conducting paths for cooling a heat generating volume. Int. J. Heat Mass Transfer 40, 799-816.

Bejan A (2020). Freedom and evolution: hierarchy in nature, society and science. Basel: Springer.

Bourdieu P (1966). "Condition de classe et position de classe." European Journal of Sociology 7, 201-223.

Dyson F (2016). "Is life digital or analog?" John Brockman, ed. Life: The Leading Edge of Evolutionary Biology, Genetics, Anthropology, and Environmental Science. New York: HarperCollins.

Domingo E, Schuster P (2016). Quasispecies. Basel: Springer.

Eigen M 1971. Self-organization of matter and the evolution of biological macromolecules. Naturwissenschaften 58, 465-523.

Hofbauer J, Sigmund K (1998). Evolutionary Games and Population Dynamics. Cambridge: Cambridge University Press.

Jacobs J, “The self-destruction of diversity," Death and Life of Great American Cities. New York: Random House, 1961: 241-257.

Landauer TK, Dumais ST (1997). "A solution to Plato's problem: The latent semantic analysis theory of acquisition, induction, and representation of knowledge." Psychological Review 104, 211-240.

Nowak MA, Komarova L (2001). "Towards an Evolutionary Theory of Language.” Trends in Cognitive Sciences 5, 288-295.

Nowak MA, May RM (2000). Virus Dynamics, Oxford: Oxford University Press.

Page K, Nowak MA (2002). “Unifying Evolutionary Dynamics.” J. theor. Biol. 219, 93-98.

Wagner A, Rosen W (2014). "Spaces of the Possible: Universal Darwinism and the Wall Between Technological and Biological Innovation.” Journal of the Royal Society Interface 11, 20131190. 


\title{
Digitization: Creative and Open-ended
}

\section{Authors}

Dan C. Baciu ${ }^{1}$

Delft University of Technology, Netherlands.

\begin{abstract}
The alphabet, the genetic code, and all of our human languages and those used by computers have something in common: They all are examples of digital systems. The main mathematical property of digital systems is that they use only a small number of symbols to create open-ended worlds of possibilities. For example, by creatively combining and recombining the fingers on one hand, digital systems can encode for countless numbers. One can be creative, count, and count, and never return to the same number. In digital systems, there typically are so many possibilities to explore that diversification becomes necessary to cover sufficient ground. Thus, creativity and diversification are consequences of digital systems. In this article, I explain how these two processes-creativity and diversification - can be modeled with replicator-mutator-equation, and how this equation describes and explains a broad range of observations in human behavior, culture, and society.
\end{abstract}

\footnotetext{
${ }^{1}$ https://orcid.org/0000-0002-0043-5616
} 


\section{Supplementary Material}

\subsection{Definition of digital system}

A digital system is defined as a system in which a small number of symbols is combined to create a larger number of new symbols. This definition of digital system is commonly used in science. It can be found for example in Nowak \& Komarova 2001. A similar definition is also given in Freeman Dyson's essay "Is Life Analog or Digital?" The essay envisions theoretical ways in which life could in principle be analog and the consequences that could arise. The essay also acknowledges that biological life commonly is digital. Dyson recounts in particular that some scientists simply assume that life must be digital, without even questioning the possibility that some yet undiscovered form of life may be analog. Dyson's essay was originally written in 2001 and published in 2016 in the volume "Life: The Leading Edge of Evolutionary Biology, Genetics, Anthropology, and Environmental Science," edited by John Brockman (Dyson 2016). The volume contains essays of leading figures in various sciences and is thus an example of common usage of scientific terms such as "digital."

\subsection{Examples of digital systems}

According to the given definition of "digital system," the alphabet must be one. It uses some thirty letters to encode for any word. Most human languages are presently written, or can be written with alphabets that are direct descendants of the Phoenician alphabet. This is true for example for languages written in the Latin, Greek, Russian, Vedic, and some East Asian scripts such as the Khmer, Thai, and Lao scripts, as well as languages written in the phonetic alphabet or with phonetic symbols. The alphabet is a fascinating digital system because it shows how adjustable digital systems can be.

Human languages are digital, as has been considered for example by Nowak \& Komarova 2001. They distinguish multiple levels at which languages are digital. First, a small set of sounds (in technical terminology known as phonemes) is combined into word roots, prefixes, suffixes, etc., which are combined into words. Then, words are combined into sentences. Finally, sentences are combined into text, which is used by people to formulate countless, diverse ideas. While some languages may have more sounds than others, the number of sounds is always limited, and these sounds are always combined to formulate countless, diverse ideas.

Human cultures are digital not only because they are partially based on language, which is most evident in literature, theater, or poetry. Digital systems can also be found in music and in the visual arts. Music is digital because it works with a limited set of musical intervals that are pressed into scales with limited number of notes. These notes are combined to render countless musical motives, songs, and entire compositions. Visual art is digital, too, because it works with a set of common formal elements and motives. Formal analysis of artworks was developed for example by Heinrich Wölfflin, one of the founding figures of modern art history. Wölfflin devised a system of classification of artistic expressions based on recurring formal elements. His book "Principles of Art History" lays down the methods of such formal analysis and is recognized as a foundational work in art history. Today, formal analysis is sometimes referred to as formalism. Through form, art communicates content. The content can sometimes be separated from the form, for example when it can be expressed as language. In this latter case, the content clearly remains digital. We have already found that languages are digital systems. One could make the case that art and other types of culture may also go beyond form or words. Art may express emotions. In psychology, it is commonly recognized that most cultures know of a limited set of emotions. Yet, emotions may be combined and recombined to create complex feelings and artistic expressions. In this case, too, art and culture remain digital.

The genetic code is yet another digital system because it is based on two base pairs. There exist slight variations, for example in DNA and RNA, but the language remains the same. Triplets of base pairs encode for amino acids, which, in turn, are combined and recombined to form sequences of amino acids that are spliced together (i.e. combined and recombined) to be expressed as proteins. On the next level of complexity, proteins are combined and recombined to facilitate more complex processes, which, in turn, lead to the specialization of cells into different types of cells, which together form larger organisms. Here, too, there is a multiple-level digital system, 
similar to the one described above for human language. Interestingly, the vocabulary of words used by an author may be similar in size to the vocabulary of proteins (proteome) their body uses, and, most importantly, in both genetic and linguistic information, there are multiple levels of digitization that work together. At the same time, the computer code used to encode for letters and words has only two symbols, it is a binary code, and the genetic code has only two base pairs. Thus, from base pairs to proteome and from binary computer code to human culture there is a "point to area" flow, which leads to hierarchies predictable through the constructal law. We observe these hierarchies in the nested digital systems, which constitutes a wholly new application of the constructal law (Bejan 1996).

Physical and chemical systems are digital system because they are based on a limited set of subatomic particles that are combined into a limited number of atoms and an open-ended number of chemicals. The genetic code mentioned before is part of this open-ended world of chemicals.

Architectural and urban systems are digital system because transparent and opaque materials are used to create a number of building elements such as doors, walls, ceilings, roofs, and fenestration, which are combined and recombined into a much larger number of building types that allow for different types of human activity. In turn different types of buildings and infrastructures such as bike lanes, sidewalks, etc. are combined to create diverse urban spaces.

Computer systems are digital systems because computer data is written in two symbols that are combined and recombined into an open-ended world of possibilities with a multi-level architecture similar to biological information and human culture.

Some digital systems have become more digital. For example, there exist more than 500 known amino acids, but only 22 are used in proteins, and only 20 are used in most organisms. "Selenocysteine" is one of the two amino acids that are rarely used (it was originally discovered by Thressa Stadtman, 1974). This amino acid is found in all three domains of life, but not in all lineages. This means that in some lineages, its usage was lost. The diversity of life has increased on Earth while the number of distinct amino acids used for folding proteins has decreased. A similar case can be made in culture. In early music, a tone was often split into quarter tones, and there was a multitude of musical scales (for example Dorian, Ionian, Lydian, Aeolic, Phrygian); today, a tone is commonly split into half tones only, and most music is based on either a major or minor scale. At the same time, the diversity of songs has increased. There's everything from Bach to present-day music in major or minor scales. Similarly, the alphabet has evolved to be written with fewer and fewer underlying signs. In computers, two signs suffice to encode for the entire alphabet and a cornucopia of special characters. At the same time, the diversity of human culture recorded in computer systems has increased.

The advantage of becoming more digitized is obvious. The fewer symbols are used to encode for information, the easier it is to reproduce this information; and reproduction is part of the replicator-mutator-equation. On the other hand, the success of the system depends on the possibilities that it opens through creativity and diversification. If a digital system has multiple levels of digitization, creativity can act at high levels. In turn, recombination of information at high levels gives life many options to diversify. It is evident then, that reproduction, creativity, and diversification as life processes may support the evolution of systems that are increasingly digital, as we observed. Life "goes digital" on its own-it turns systems into multi-level digital systems in which easy encoding makes reproduction is easy, while creativity can operate at high levels, and diversification has many ways to go.

After considering so many digital systems, we must also admit that digitization and the replicator-mutator-equation go hand in glove. They mutually enforce each other: Digitization begins with a small number of symbols that are combined and recombined to create an open-ended world of possibilities. This opens up the path to creativity and diversification. On the other hand, creativity and diversification make most sense in the presence of reproduction, or else good information cannot spread. This leaves us with the three processes-reproduction, creativity, and diversification - which enter the replicator-mutator-equation. Yet, we also know that the replicator-mutator-equation works best when reproduction is easy, creativity acts at high levels, and diversification has many ways to go, and these requirements end up making digital systems even more digital. 


\section{Mathematical models}

The replicator-mutator-equation states that new culture is a product of all old culture, creativity, and diversification. These three parts are discussed below in more detail. The Quasispecies equation and Lotka-Volterra equations are special cases of the replicator-mutator-equation (Page, Nowak 2001). These facts speak to the general validity of our model.

The statement that new culture is a product of old culture is the same as saying that new culture is the "reproduction" of old culture. In human culture, reproduction occurs whenever a message is transmitted from a sender to a recipient. Claude Shannon defined communication as the "reproduction" of a message at a new place (Baciu 2019). In biological life, reproduction is commonly present and recognized as a key process of life. Thus, there can be no doubt that reproduction is common to all life, cultural and biological. We must consider reproduction to get realistic models.

Creativity is different from reproduction in that it makes changes to the information that is reproduced. Creativity can thus also be defined as "approximate reproduction." Through creativity, evolutionary adaptation becomes possible. In biology, creativity is abundant. The reproductive enzymes of cells creatively engage with genetic information, often reproducing this information with slight changes. Clearly, when changes are made, genetic information is not only exactly but also approximately reproduced. Common changes during genetic reproduction are mutations, deletions, duplications, and recombination. In the 1970s, Manfred Eigen proposed to mathematically model exact and approximate reproduction as parallel channels of reproduction. This approach allowed him to develop the quasispecies equation for molecular evolution. Using the quasispecies equation, Eigen and his followers were able to predict frequency distributions of genetic variants in evolving populations of bacteria. The quasispecies equation has also been used in virus dynamics. It is commonly known today that viruses evolve in quasispecies of virus, which makes it possible to develop vaccines. A quasispecies of variants is sometimes imagined as a creative cloud of variants. The point is that the variants in a quasispecies are close together, in a finite cloud, rather than widely or chaotically dispersed over the space of genetic possibilities. Therefore, a vaccine only needs to cover all existing variants in a given cloud. More generally, the quasispecies equation is a mathematical formulation of a "variation-selection process." The equation is now featured in many textbooks of evolutionary dynamics as well as in a dedicated monograph, published in 2016 (Domingo, Schuster 2016).

In my 2017 report (Baciu 2017), I presented an equation that models collective creativity in human culture, deriving it from basic premises about communication. This equation is equivalent to the quasispecies equation. I used my formula to study human culture, predicting frequency distributions for variants of a given term in a given public discourse; and I tested my predictions against empirical evidence. The tests confirmed the empirical validity of the theory. I also recognized that the frequency distributions often predicted through the quasispecies equation come close to power laws, which are a class of frequency distributions commonly found in human culture (as well as in biological life). Power laws have been known for a long time. Vilfredo Pareto is credited to have discovered them in the late $19^{\text {th }}$ century in economics; and they are therefore also termed "Pareto distributions." Power laws are consistently obtained as empirical results in the study of human culture and economy. They were obtained after Pareto by Felix Auerbach, Alfred Lotka, Derek de Solla Price, Adrian Bejan (Bejan 2021), and countless more researchers. Thus, my testing is based on robust empirical evidence. Such evidence can be gained from the study of almost any corpus of text. Take any corpus of text in natural language, divide it into words (by splitting over white space and punctuation), count word frequencies, and you will likely receive something close to a power law. Of course, my testing was a little more complex, as can be seen in the articles.

The equation of collective creativity that I proposed in 2017 can also be connected to Shannon's work on communication. When Shannon defined communication as the "reproduction of a message at a new place," he envisioned the possibility that noise interferes during communication. He thus spoke of "exact reproduction," which does not involve noise, and "approximate reproduction," which does. In my 2019 publication (Baciu 2019), I showed that my equation of 2017 can be obtained from Shannon's framework if this framework is expanded in two important ways: First, multiple acts of communication must be assumed to occur in parallel (multiple people can talk to each other at the same time). As a consequence, exact and approximate reproduction become parallel channels. As mentioned before, the quasispecies equation, too, models exact and approximate reproduction are parallel channels. This means that Shannon's framework becomes equivalent to the 
quasispecies equation in its most general form. A second step is necessary to obtain the actual equation of creativity that I proposed in 2017. This step is equally straightforward. All needed is considering that approximate reproduction may occur as a result of human creativity rather than through the interference of noise alone. This consideration generalizes Shannon's definition of communication to also include creativity, making it apply to cultural evolution.

Mathematically in the replicator-mutator-equation, the effects of exact and approximate reproduction, as parallel channels of reproduction, are expressed through the term $q_{i j}$, which records the probability that a given variant $i$ is transformed into variant $j$. The two subscripts $i$ and $j$ are indices, and they can happen to stay for different variants or for one and the same variant. When $i$ and $j$ are the same variant, we speak of exact reproduction. When $i$ and $j$ are different variants, we speak of approximate reproduction. The various exact and approximate reproduction rates recorded by various $q_{i j}$-values can also be represented as a matrix $Q$ that contains all such values. The matrix $Q$ contains all rates of reproduction, exact and approximate. Sometimes, the matrix representation feels more intuitive, but it really is mathematically equivalent. The quasispecies equation is given below.

Quasispecies equation. The quasispecies equation differs from the replicator equation through the term $q_{i j}$, the probability that variant $i$ is reproduced as variant $j$, also representable as matrix $Q$, which is the creativity matrix.

$$
\dot{x}_{i}=\sum_{j=1}^{n} x_{j} f_{j} q_{j i} .
$$

The mathematical modeling of exact and approximate reproduction as parallel channels applies to multiple known settings: Approximate reproduction can occur due to genetic mutation and recombination, it can occur through the interference of noise, or it can occur through human creativity. The distinction between genetic creativity, noise, and human creativity is that approximate reproduction in the tree settings is shaped by different mechanisms: Genetic creativity is primarily shaped by the types of creativity that typically occur during genetic reproduction. On the other hand, noise, as in Shannon's case, primarily comes from technical problems. Finally, human creativity is primarily shaped by the brain's typical ways of making associations. Thus, the matrix $Q$ can be either a "mutation matrix," a "recombination matrix," a "noise matrix," or an "association matrix." With "association matrix" I refer to a matrix that represents the associations that the brain makes between words or between ideas. Mutation matrices, recombination matrices, and association matrices are all "creativity matrices" in that they help populations of living organisms be collectively creative and adapt to physical circumstances.

If the creativity matrix $Q$ is known, it is possible to calculate frequency distributions in the population, but the matrix may be most useful because it records how creativity occurs. Each value in the matrix tells how frequently creativity transforms one given variant into another. Let us consider the simple case in which there is only reproduction and creativity. If reproduction and creativity occur over may generations, the effect is that only the most successful quasispecies survives (one can also imagine this as one group of interrelated variants or as one creative cloud of variants), while all other quasispecies are pushed into extinction through competitive exclusion. This is not the only empirical result that can be obtained. If, we look at our data and find multiple quasispecies that are independent of each other, we have to deal with diversification.

Diversification occurs in countless biological and cultural systems. For example, distinct animal species are independent evolutionary units. This is true because genetic mutation and recombination do not transform distinct animal species into each other. Example: We never observe chicken become foxes. Thus, diversification cannot occur through reproduction and creativity alone. Diversification must occur through interplay.

Mathematically, interplay between variants is represented through the term $f_{i}(x)$, which is a function of $x$, the frequency distribution of variants in the population. Dependent on how many copies of each variant exist, the interplay between variants may have different effects on the reproduction rate of any variant $j$. Example: if there are many chickens somewhere, and if some foxes can pray on them, then, as a result of this interplay, one can expect to soon see many more foxes.

Technically speaking, the effects of the interplay depend on $x$, which is the frequency distribution of variants (number of chickens and foxes), and they depend of $f_{i}$ which is the nature of the interplay itself (praying). To 
stress the importance of $x$, the interplay between variants is also termed "frequency dependent selection." Nevertheless, both terms are relevant. The full replicator equation is given below.

Replicator equation. The replicator equation differs from the quasispecies equation through the term $f_{i}(x)$, which accounts for frequency dependent selection.

$$
\dot{x}_{i}=x_{i} f_{i}(\mathbf{x}) .
$$

Diversification must involve the term $f_{i}$. That's because diversification cannot take place through creativity alone; some interplay between variants must take place. In addition, diversification must involve the frequency distribution $x$. That's because diversification can only occur if the reproduction rates of multiple species are the same, in the long run. If this were not the case, competitive exclusion would apply. One species would outcompete all others. Even if the environment were diverse, from the beginning, the one species that most efficiently reshapes the environment would outcompete all others. Example: This coevolution of species and environment is believed to have occurred when photosynthesis was discovered several billion years ago. Photosynthesis transformed the atmosphere from a $\mathrm{CO}_{2}$-rich to an $\mathrm{O}_{2}$-rich atmosphere, benefiting only the discoverers. We must conclude that the environment may evolve with the species, and that the reproduction rates must be the same for multiple species, in the long run. Now, if the reproduction rates are the same in the long run, it means that the populations react to each other's size - and this is exactly frequency dependent selection. Example: No predatory populations can exist without prey populations, and the sizes of the two depend on each other. This means that the frequency distribution $x$ must be considered. We remain with the term $f_{i}(x)$. Diversification must occur through a process recorded through this term. The contrary is not true. Not all processes recorded by $f_{i}(x)$ lead to diversification, but diversification is maybe the most important outcome because it allows evolution to search in multiple directions. Through diversification, there are multiple creative units that can evolve into multiple independent directions.

Diversification is most closely linked to one particular type of interplay between variants, namely the interplay between prey and predatory species. This type of interplay is modeled through Lotka-Volterra equations. Mathematically, these equations can be generalized to obtain the generalized Lotka-Volterra equations, which are mathematically equivalent to the replicator equation, which de facto is the term $f_{i}(x)$ in the replicator-mutatorequation (Hofbauer, Sigmund 1998; Page, Nowak 2001).

Some authors make a distinction between intra-species evolution, which takes place within one evolutionary unit, and interspecies evolution, which takes place between the units. Clearly, the term $q_{i j}$ accounts for intraspecies evolution, while the term $f_{i}(x)$ accounts for interspecies evolution. Interspecies evolution is sometimes termed ecology. In addition, the SIR-model of epidemiology and its variant formulations are related to LotkaVolterra equations (Nowak, May 2000). This is further testimony to the generality of our mathematical models.

In biological life, diversification takes place almost everywhere. Most animals live in diverse ecosystems. Competitive exclusion does often not take place. Instead, the diversity of living beings in an ecosystem is supported by their ecological interplay, and most importantly by prey-predator interactions, as mentioned before. It is true that, the co-existence of animals in ecosystems is also supported by the diversity of the habitat itself, but the habitat is partly created by the species that live in it, as we have already observed. Furthermore, species do not live each isolated in its own region, but they interact and grow out of centers of ecological diversity. Therefore, the source of diversity is inextricably linked to the species themselves rather than the diversity of their habitat. Prey-predator interactions remain the main cause of diversification. This insight is commonly known and plays an important role for example in ecosystem management. A broadly known case is Yellowstone National Park. The introduction of wolfs as predatory animals has helped the park recover some of its original ecological diversity. When the wolfs were introduced, they prayed on the most frequent animals, while letting rare animal species recover. This led to an increase in ecosystem diversity. Yellowstone is no exception. In absence of predators, it is customary that the most successful prey species must be diminished in number by human hunters, while rare species must be protected. Where the predators are gone, the humans must take the role of predators to preserve diversity. This knowledge serves in determining the number of animals, for example deer, that are hunted in fall. If, on the other hand, predators do not hunt primarily on the abundant species, they will not help the ecosystem stay diverse. This is the case in which $f_{i}(x)$ does not lead to diversification. Territorial development as practiced by agrarian societies has had exactly this effect. Ecosystem diversity is decreasing today because 
agrarian societies take a piece of land, removing it from both frequent and rare species. Ecosystem diversity is not supported by this activity because multiple species are killed indiscriminate of their rarity while habitat size is overall decreased. Another path to extinction is preying on the rare species. This occurs for example if some rare furs and feathers are praised for their rarity. Then, there is an incentive to hunt and decimate rare species, which may lead to extinction.

On the other hand, prey-predator interaction in which the predators hunt the most abundant species may not only support diversity but also lead to the evolution of wholly new species, which evolve to escape predation. Escape variants may escape predation each in its own way, which may lead to speciation in multiple directions. Hence, there is additional diversification. This phenomenon can be observed for example in the interplay between immune system and persistent viral infections. The immune system "preys" on the virus, which mutates in multiple directions and evolves into diverse escape variants (Nowak, May 2000).

In human culture, diversification is equally abundant. Almost all human cultures are diverse. There are always diverse types of activities that humans do, and there are always diverse ideas that humans share. Clearly, diversification must somehow take place. Logically speaking, diversification must occur through processes representable through the same term $f_{i}(x)$. Could it be that diversification in human culture also occurs through something equivalent to prey-predator interaction? If so, what then are the predators of human culture?

There was long an intuition that some or all ideas in human culture spread like viruses. People sometimes speak of "viral news" that come and go like waves - and fashions are also known as "fashion waves." In my 2018 work (Baciu 2018), I have predicted that fashions, news, and other culture come, go, and return in waves as a result of the interaction between the spread of news and the spread of human habituation to the news. The idea is simple: First, the news spread, then human boredom spreads. This means that the news is the prey, while boredom is the predator. The setup results in equations equivalent to Lotka-Volterra equations (Baciu 2018). Through these equations, I was able to predict and empirically show the existence of not only fashion waves (Baciu 2018, 2019), but also entire cycles of cultural diversification (Baciu 2020).

During the cycles of diversification, a recurrent pattern is found. Growth leads to loss of diversity, while recession allows for diversification to return and catalyze new growth. Such cycles are empirically known in urbanism (Baciu, Birchall 2021). Decades ago, famous urban activist Jane Jacobs termed them "self-destruction of diversity" (Jacobs 1961). The point made here is that these cycles emerge out of the replicator-mutator-equation because the replicator and mutator parts of the equation tend to fall apart in their working, which results into phases of diversification and phases of growth.

It is unknown whether cycles of growth and diversification occurred in animal evolution. It is possible that they did. The fossil record has long phases of stability interrupted by shorter phases of reorganization. The phenomenon is also known as "faunal succession." These cycles of stability and reorganization could be cycles of growth and diversification. Growth is stability, while diversification is reorganization.

\section{Creativity and diversification as analyzed through the latest wave of digital tools}

Dimensionality reduction is a common type of data analysis that is used in many disciplines. Pierre Bourdieu used it to detect "social fields," and dimensionality reduction is also used in text analysis, image analysis, and beyond. The common outcome of dimensionality reduction is a set of groups of interrelated items. In sociology, Bourdieu called such groups social fields. In the humanities, they are called topics, sometimes genres.

The mathematical tools to perform dimensionality reduction have themselves evolved. In psychology and linguistics, Landauer and Dumais (1997) developed Latent Semantic Analysis, which is a type of dimensionality reduction. There had been long doubts whether the results from dimensionality reduction are meaningful or not. Some researchers believed that the results only "seemed" meaningful to human interpreters because human interpreters can make sense of anything whatsoever. This was proved wrong by Landauer and Dumais, as they showed that the analysis could be used to predict synonyms. (One can take multiple words, only two of which are synonyms, and one can use dimensionality reduction to predict which two words are the synonyms.) The dimensions obtained from dimensionality reduction were better predictors of synonymy than the same data 
before dimensionality reduction. An entire generation of computer scientists followed who improved dimensionality reductions by testing how well they worked for synonymy prediction. Nevertheless, the question remained open why those dimensionality reductions were useful at predicting synonyms, to begin with.

Landauer and Dumais understood that the first step in their processing (an algorithm called Term Frequency Inverse Document Frequency or tf-idf) left them with word-association matrices. From their data-driven work, they also knew that these association matrices had to be further processed through matrix decomposition to improve on the synonymy prediction. The matrix decomposition was the type of dimensionality reduction that gave them the best testing results. The problem was that they did not know, theoretically, why this latter step was necessary. To answer this theoretical question, Landauer and Dumais attempted to develop a theory of learning which, as they explained, had no precedents. Only few years after Landauer and Dumais, two other scientists Nowak and Komarova (2001) proposed that some aspects of natural language may be modeled with the replicator-mutator-equation. They studied how arbitrary signs may evolve to signify arbitrary content, but their main research interest with respect to the replicator-mutator-equation was the evolution of grammatical rules. Interestingly, in their case, the $f_{i}(x)$ term is postulated to have values that lead to the opposite of diversification. Nowak and Komarova were primarily interested in the long run of language acquisition from parents to children, but they did not study word frequency distributions.

In my 2020 article, I have explained why the matrix decomposition method employed by Landauer and Dumais simulates exact and approximate reproduction, as it is done through the quasispecies equation, and therefore through the term $q_{i j}$ in the replicator-mutator-equation (Baciu 2020). The matrix decomposition empirically chosen by Landauer and Dumais is mathematically equivalent with simulating evolution through the term $q_{i j}$. Given that the empirical result obtained by Landauer and Dumais has been replicated countless times, the empirical support for my theoretical proposition is as solid as it only can be. Bringing together theory and empirical observation allowed me to understand how exactly creativity works in language, namely as played out on association matrices (associations between words, or associations between ideas, etc.). Such association matrices can be obtained for example through the method already used by Landauer and Dumais. Association matrices are creativity matrices $Q$.

Bringing together theory and empirical analysis allowed for much more. Most importantly, the matrix decomposition method employed by Landauer and Dumais does not reveal the existence of only one group of synonyms but some 200-500 groups or more, dependent on the quantity of text that is processed (Landauer, Dumais 1997). This result, too, has been reproduced countless times. Clearly, we deal here with diversification. The same phenomenon can also be seen through the lens of research on "associative triplets." Not all words evoke equally many and equally tight associations, rather the world of words is distributed in diverse groups of words. On second consideration, this is not surprising. Many schools of writing teach that writers should avoid needlessly repenting the same words. The more a word is used, the more one should avoid using it. This means that there is an interplay in many a writer's brain between repeated words, and words that should no longer be repeated: First there is a word, then there is a tendency to repeat the word, and then there is an active wish not to repeat that word, and this wish increases the more the word has actually been repeated. Here again, we have LotkaVolterra interaction and diversification. In the end, writers who avoid repeating the same words will broaden their vocabulary, and we have diversification describable through the term $f_{i}(x)$. Habituation, boredom, and the wish to avoid repetition can be seen as the predators of human culture. They are equally important as regular ideas.

Dimensionality reductions have also been applied to sociology and linguistics, as well as other fields of study. They are commonly known to give meaningful results, and almost everywhere, they reveal diversity rather than unity. I went on to apply dimensionality reductions to yet another field to further test my conclusions. I chose culture, developing a method to decode cultural content, map it, and study its historical trace. I looked in particular at names and terms. As can be seen in my 2020 article, names and terms tend to cluster in groups distinct of those formed by regular words (In the left chart of the following interactive illustration, there is little data along the diagonal: https://dan-baciu.github.io/metadata-7D/?type=TimeD\&left $Y=0 \& l e f t X=1$ ). Words go with words, while cultural content goes with cultural content. Thus, cultural studies remain a domain distinct from linguistics. On the other hand, both culture and languages evolve through the same processes that can be modeled with the same tools, as my article has showed. This allows me to formulate a theory of life of broad generality. 
What makes cultural content interesting, in particular, is that it has a spatiotemporal trace to it: Culture has geographies and histories, which I analyzed. In ecology, habitat diversity can be an initial cause of diversification; although animal species can shape their habitat, and therefore, diversification in the long run depends on the interplay between species. Following these considerations, it seems relevant to test whether diversification in human culture is primarily an effect that comes out of the diversity of geographical settings. If this were true, each diverse group of ideas would have to be associated with exactly one geographical area. However, my paper rejects this hypothesis (Baciu 2020: Fig. 2). As evident from my research, diverse groups of ideas can be associated to similar geographies. This means that diversification, at least in those cases, evolves though the interplay between variants rather than through adaptation to distinct geographies. Diversity grows out of oases of cultural interplay.

It is also the case that multiple groups of ideas can be associated with the same period of time, which shows that diversification again evolves through the interplay between variants even during one and the same period of time (Baciu 2020: Fig. 3). Here again, interplay between groups of ideas in its most general form can be represented through the term $f_{i}(x)$, and we have another empirical proof that the effects of creativity and diversification can be modeled through the replicator-mutator-equation.

\section{Broader Significance of Theory and Observations}

A large body of research across science and humanities has come to deal with creativity and diversity, which, as scientific concepts, proved immensely relevant in understanding anything from nature and ecosystems to cities and culture. In my research, I have developed mathematics and theory to explain and predict diversification processes. My work allows researchers to study creativity and diversification through the same mathematics, theories, and digital tools, regardless of their field of study. And through my research, insights from one field have become applicable to the others. This is because all of these fields of study, from the life sciences to the humanities and the sciences of the city, deal with life in its creativity and diversity, and in all of these fields, life is based on digital systems, as I presented them in this present article.

In addition, my work may occasionally reach beyond science. The $21^{\text {st }}$ century is a century of digitization and globalization. It is now time both for more accurate prediction and for a new worldview. After all, we are a global, digitized society of unprecedented scale. My work provides a theory of digitization that may be broad enough to serve as a basis for an inclusive, and digitally informed worldview. 


\section{Easy access to articles and datasets}

Baciu DC, Callum B (2021). "Mapping Diversity: From Ecology and Human Geography to Urbanism and Culture." OSF preprints. https://doi.org/10.31219/osf.io/sdvaz

Baciu DC (2020). "Cultural life: Theory and empirical testing." Biosystems 197.104208. Article publicly available at: https://doi.org/10.1016/j.biosystems.2020.104208

Baciu DC (2019). "Chicago Schools: Large-scale Dissemination and Reception." Prometheus 2: 20-43. https://escholarship.org/uc/item/22v9g5m

Data processed with geocodes, visualized. Publicly available: https://doi.org/10.25496/W2RP4H

Data processed with publisher names, visualized. Publicly available: https://doi.org/10. 25496/W2WC7S

Data processed with historical information, visualized. Publicly available: https://doi.org/10.254 96/W2159Q

Google ngrams (2012) publicly available: https://storage.googleapis.com/books/ngrams/books/datasetsv3.html

\section{Bibliography}

Baciu DC (2015). "Systemic Analysis of Cooperation: Architects, Urban Space and Tourism.” JETK 4, 27-30.

Baciu DC (2016). "Sigfried Giedion: Historiography and History of Reception on a Global Stage."

Ar(t)chitecture, Haifa: Technion Israel Institute of Technology, 40-52.

Baciu DC (2017). “The Chicago School: Evolving Systems of Value.” Report, HathiTrust Research Center.

Baciu DC (2018). "From Everything Called Chicago School Towards the Theory of Varieties." Doctoral dissertation, Illinois Institute of Technology.

Baciu DC (2019). "The Chicago School: Large-Scale Dissemination and Reception." Prometheus 2, 20-41.

Baciu DC (2020). "Cultural Life: Theory and Empirical Testing." Biosystems 197.104208.

Baciu DC (2021). "Is "Cultural Life" a Form of Life?" Lecture, TU Delft, published in: Igloo Feb. 2021, 5861.

Baciu DC, Birchall C (2021). "Mapping Diversity: From Ecology and Human Geography to Urbanism and Culture." OSF preprints.

Bejan A (1996). Constructal-theory network of conducting paths for cooling a heat generating volume. Int. J. Heat Mass Transfer 40, 799-816.

Bejan A (2020). Freedom and evolution: hierarchy in nature, society and science. Basel: Springer.

Bourdieu P (1966). "Condition de classe et position de classe." European Journal of Sociology 7, 201-223.

Dyson F (2016). "Is life digital or analog?" John Brockman, ed. Life: The Leading Edge of Evolutionary Biology, Genetics, Anthropology, and Environmental Science. New York: HarperCollins.

Domingo E, Schuster P (2016). Quasispecies. Basel: Springer.

Eigen M 1971. Self-organization of matter and the evolution of biological macromolecules. Naturwissenschaften 58, 465-523.

Hofbauer J, Sigmund K (1998). Evolutionary Games and Population Dynamics. Cambridge: Cambridge University Press.

Jacobs J (1961). “The self-destruction of diversity," Death and Life of Great American Cities. New York: Random House, 241-257.

Landauer TK, Dumais ST (1997). "A solution to Plato's problem: The latent semantic analysis theory of acquisition, induction, and representation of knowledge." Psychological Review 104, 211-240.

Nowak MA, Komarova L (2001). “Towards an Evolutionary Theory of Language.” Trends in Cognitive Sciences 5, 288-295.

Nowak MA, May RM (2000). Virus Dynamics, Oxford: Oxford University Press.

Page K, Nowak MA (2002). “Unifying Evolutionary Dynamics.” J. theor. Biol. 219, 93-98.

Wagner A, Rosen W (2014). "Spaces of the Possible: Universal Darwinism and the Wall Between Technological and Biological Innovation." Journal of the Royal Society Interface 11, 20131190. 